\title{
Differential Response of QPM, Hybrid and Composite Maize Cultivars to INM Schedules
}

\author{
Ananya Chakraborty*, Sritama Biswas, Rajarshi Banerjee, \\ Pintoo Bandopadhyay and Srijani Maji
}
Department of Agronomy, Bidhan Chandra Krishi Viswavidyalaya, Mohanpur-741252, Nadia, West Bengal, India

*Corresponding author

\section{A B S T R A C T}

\section{Keywords}

Maize, cultivars, INM, growth, yield, harvest index, economics.

\section{Article Info}

Accepted:

20 August 2019

Available Online:

10 September 2019
To understand the performance of QPM, hybrid and composite maize to rationalized Nitrogen nutrition, a two year experiment was conducted in the rabi season of 2017-2018 and 2018-2019 at Mondouri experimental farm, Bidhan Chandra Krishi Viswavidyalaya, Nadia, West Bengal, situated at $22^{\circ} 56^{\prime} \mathrm{N}$ latitude, $88^{\circ} 32^{\prime} \mathrm{E}$ longitude. It was laid in split plot design with three cultivars in the main plot - HQPM4 (Quality Protein Maize), Shresta (single cross hybrid) and NAC 6004 (composite variety); the sub plots had 6 nutrient schedules in i) $\mathrm{T}_{1}$ : control, ii) $\mathrm{T}_{2}: \mathrm{RDF}$, iii) $\mathrm{T}_{3}: \mathrm{RDN}_{75}+$ Vermicompost @ $2 \mathrm{t} / \mathrm{ha}$, iv) $\mathrm{T}_{4}: \mathrm{T}_{3}+$ Azotobactor @ 2kg/ha, v) $\mathrm{T}_{5}: \mathrm{T}_{4}+\mathrm{Zn}$, vi) $\mathrm{T}_{6}: \mathrm{RDN}_{50}+$ Vermicompost @ $2 \mathrm{t} / \mathrm{ha}$ + Azotobactor @ 2kg/ha + Zn. A recommended fertilizer dose of 180:80:80 kg NPK ha ${ }^{-1}$ of which $\mathrm{P}, \mathrm{K}$ and $20 \% \mathrm{~N}$ were applied as basal dose and remaining dose of $\mathrm{N}$ was applied as top dressing splits $25 \%$ at $1^{\text {st }}$ top dressing at 4 leaf stage, $30 \%$ as $2^{\text {nd }}$ top dressing at 8 leaf stage, $20 \%$ as $3^{\text {rd }}$ top dressing at tasselling stage and 5\% was top dressed at the grain filling stage. Vermicompost @ 2 tonnes/ha as per treatment were applied and $\mathrm{ZnSO}_{4} @ 20 \mathrm{~kg} / \mathrm{ha}$ was applied in the soil 3 days before sowing. Among three maize cultivars hybrid Shresta may be recommended for the eastern plains and HQPM 4 also enjoyed good production potential. It is also concluded that inorganic fertilizer $\mathrm{N}$ can be discounted to the extent of $25 \%$ and $\mathrm{RDN}_{75}+$ Vermicompost @ 2 t/ha + Azotobactor@ $2 \mathrm{~kg} / \mathrm{ha}+\mathrm{Zn}$ with respective economics of 1.86. The economics favoured hybrid maize, Shresta with a value of 2.1 BCR.

\section{Introduction}

Consumed by billions of people through the millennium, cereals cater the key sustenance in most of the diets. Cereals are grown in over $73 \%$ of the total world harvested area and contribute over $60 \%$ of the world food production providing dietary fibre, protein, energy, minerals, and vitamins required for human health (Das et al., 2012). Of the approximately 2.3 billion tonnes of cereals currently produced, roughly 1 billion tonnes is destined for food use, 750 million tonnes is employed as animal feed, and the remaining 500 million tonnes is processed for industrial use, used as seed or wasted (FAO 2013). With the rice posing itself as a water guzzler with reported lower water productivity of 0.25 
$\mathrm{kg} / \mathrm{M}^{3}$ in summer, wheat having a value of $0.417 \mathrm{~kg} / \mathrm{M}^{3}$ and Maize (summer) having a value of $0.363 \mathrm{~kg} / \mathrm{M}^{3}$ (Kumari et al., 2017). But weather variability induced increment of average winter temperature, reported decline in wheat productivity (Mukherjee et al., 2019). This leaves maize gaining prominence in Indian subcontinent. Maize, a C4 plant, enjoys more efficient photosynthates and also offers more value addition for nutriment making it the champion of industries, more gender compatible and serving as human and animal food as compared to wheat and rice. Maize also finds application in industry in a host of non-food applications (Murdia et al., 2016).

Since independence, the researchers are in the process of breeding composites, hybrids and reported quality protein maize cultivars in the recent past. Incapability of desirable characteristics fixation and yield gap posed difficulty in mass cultivation of composite maize which afterwards became restricted within some tribal pockets and sporadic groups of people where animal feed from maize also prevails. This made hybrid maize more popular. To address the protein malnutrition among children in the developing countries, breeding for enhanced protein content in maize resulted in advent of Quality Protein Maize (Prasanna et al., 2001) while also improving its agronomic and consumer characteristics (Gunaratna et al., 2019).

Maize being a heavy feeder of nutrients, nitrogen in particular and affordable farmers having propensity to practise indiscriminate fertilizer use, which add to pollution through losses in soil and water, create a potential health hazard. Poor framers using suboptimal fertilizer level also harm the environment through soil mining. Use of renewable and non- renewable nutrient not only improves the physico-chemical characteristics and fertility of soil but also increase the crop yields by enhancing the efficiency of applied nonrenewable sources (Lal and Shing, 1998) which emerged with the integrated plant nutrient management concept. The use of renewable resources and inputs is one the fundamental principles of sustainable agriculture that enables maximum crop productivity and minimal environmental risk incorporating biological fertilizer (Kizilkaya, 2008). The information on growth and yield of maize cultivars across composite, hybrid and QPM and their comparison becomes important to understand the issues of allocation of nutrients, through both sustainable and inorganic formats. This work has been tried to present, comprehensively, the nitrogen nutrient issue of different types of maize growers in the country towards more rationalised nutrient schedules and lower wastage of nitrogen to reduce costs.

\section{Materials and Methods}

The experiment was framed during rabi season of 2017-2018 and 2018-2019 at Mondouri experimental farm, Bidhan Chandra Krishi Viswavidyalaya, Nadia, West Bengal,

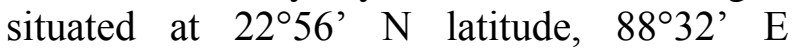
longitude falling under New Alluvial Zone of West Bengal enjoying sub-tropical humid climate with short and mild winter. The location underwent mean annual rainfall of $1457 \mathrm{~mm}$ skewed between June to September, the mean monthly temperature ranges from $10^{\circ} \mathrm{C}-37^{\circ} \mathrm{C}$. The experimental soil comes under the order of Entisol in the USDA modern taxonomical classification with sandy loam in texture consisting of $35.5 \%$ clay, $39.7 \%$ silt, and $24.8 \%$ sand with a bulk density of $1.40 \mathrm{~g} / \mathrm{cc}(0-15 \mathrm{~cm}$ depth of soil), almost neutral $\mathrm{pH}$, good drainage capacity and low available $\mathrm{N}$ and $\mathrm{P}$, and medium organic carbon as well as K status. Standard analytical procedures were followed for carrying out the chemical analysis of soil samples (Jackson, 1973). 
The experiment was conducted in split plot design and replicated thrice, where the main plot treatments comprised maize cultivars in i) $\mathrm{V}_{1}$ : HQPM4 (QPM hybrid maize), ii) $\mathrm{V}_{2}$ : Shresta (Single cross hybrid) and (iii) $\mathrm{V}_{3}$ : NAC 6004 (Composite variety); in the sub plot there was 6 nutrient schedules in i) $\mathrm{T}_{1}$ : control, ii) $\mathrm{T}_{2}: \mathrm{RDF}$, iii) $\mathrm{T}_{3}: \mathrm{RDN}_{75}+$ Vermicompost @ 2 t/ha, iv) $\mathrm{T}_{4}$ : $\mathrm{T}_{3}+$ Azotobactor @ 2kg/ha, v) $\mathrm{T}_{5}$ : $\mathrm{T}_{4}+\mathrm{Zn}$, vi) $\mathrm{T}_{6}: \mathrm{RDN}_{50}+$ Vermicompost @ 2 t/ha+Azotobactor@2kg/ha + Zn.

A recommended fertilizer dose of 180:80:80 $\mathrm{kg}$ NPK ha ${ }^{-1}$ of which P, K, 20\% N were applied as basal dose and remaining dose of $\mathrm{N}$ was administered in the following manner as top dressing viz. $25 \% \mathrm{~N}$ applied as $1^{\text {st }}$ top dressing at 4 leaf stage, $30 \%$ as $2^{\text {nd }}$ top dressing at 8 leaf stage, $20 \%$ as $3^{\text {rd }}$ top dressing at tasselling stage and 5\% was top dressed at the grain filling stage.

The required quantity of vermicompost @ 2 tonnes/ha as per treatment were applied for each respective plots a day before sowing, on the soil surface and mixed into the soil. Soil application of $\mathrm{ZnSO}_{4} @ 20 \mathrm{~kg} / \mathrm{ha}$ was done 3days before sowing. The growth attributes involved final plant height, dry matter accumulation and LAI (Watson, 1947) taken during peak growth stage.

Associated characters recorded included length and girth of cobs. Yield and yield attributing characters included data on no. of cobs per plant, grains per cob, test weight, shelling $\%$ and harvest index. Quality attributes analyzed were crude protein content as reported in FAO, 2003 and carbohydrate content.

The statistical analysis of the data generated during investigation was carried out on computerized system i.e OP Stat Statistical Software Package for Agricultural Research (Sheoran et al., 1998).

\section{Results and Discussion}

\section{Growth attributes}

Table1. shows that plant height in 100 DAS for varietal means were significant in both the years (2017-18 and 2018-19) with hybrid maize type scoring the best with $225.72 \mathrm{~cm}$ in $1^{\text {st }}$ year and $241.29 \mathrm{~cm}$ in $2^{\text {nd }}$ year both being at par with the HQPM4 and significantly superior over the NAC 6004 maize cultivar. The 100 DAS data for shoot weight, along with values for dry cobs, at that stage, had Shresta scoring the maximum in both years with $1008.35 \mathrm{~g} / \mathrm{m}^{2}$ in $1^{\text {st }}$ year and 1083.97 $\mathrm{g} / \mathrm{m}^{2}$ in $2^{\text {nd }}$ year. HQPM4 also had a pronounce growth comparative to the NAC 6004 with the value of $998.11 \mathrm{~g} / \mathrm{m}^{2}(2017-18)$ and $1079.96 \mathrm{~g} / \mathrm{m}^{2}(2018-19)$ and both of the hybrid varieties were significant over the NAC 6004 maize type $\left(880.00 \mathrm{~g} / \mathrm{m}^{2}\right)$ in $1^{\text {st }}$ year as well as in $2^{\text {nd }}$ year $\left(943.36 \mathrm{~g} / \mathrm{m}^{2}\right)$.

Among the $\mathrm{N}$ management schedules $\mathrm{RDN}_{75}+$ Vermicompost+ Azotobactor and $\mathrm{ZnSO}_{4}$ application resulted in maximum and significant plant height $(235.27 \mathrm{~cm}$ in $2017-18$ and $255.03 \mathrm{~cm}$ in 2018-19), dry matter accumulation $\left(1092.18 \mathrm{~g} / \mathrm{m}^{2}\right.$ in $2017-18$ and $1192.67 \mathrm{~g} / \mathrm{m}^{2}$ in $2018-19$ ) and LAI of 3.27 in the $1^{\text {st }}$ season and 3.41 in the $2^{\text {nd }}$ season. Similar increasing dry matter in maize with $\mathrm{N}$ nutrition has been reported by Ram et al., 2009, in conjunction with biofertilizers and organics by Savalgi and Savalgi, 1992 and by zinc supplementation by Arya and Singh, 2000. The improvement in LAI values as a response to organic sources in $\mathrm{N}$ management has been previously reported by Kumar et al., 2008.

\section{Yield associated characters}

Length of cob data revealed the maximum cob length of hybrid maize was $16.11 \mathrm{~cm}$ keeping at par values with QPM $(15.85 \mathrm{~cm})$ and higher 
than NAC $6004(14.84 \mathrm{~cm})$ in the $1^{\text {st }}$ year. In the $2^{\text {nd }}$ year, hybrid Shresta also emerged significantly higher with $16.92 \mathrm{~cm}$ than NAC $6004(15.14 \mathrm{~cm})$ cob length. Among the various nitrogen management schedules $\mathrm{RDN}_{75}+$ Vermicompost+ Azotobactor and $\mathrm{ZnSO}_{4}$ application registered significantly higher mean cob length of $16.61 \mathrm{~cm}$ and 17.18 $\mathrm{cm}$ in the successive years. Among the three varietal means of cob girth, hybrid Shresta proved best with a value of $13.04 \mathrm{~cm}$ (2017-
18) and $13.46 \mathrm{~cm}$ 2018-19) which were statistically at par with HQPM4. In both the seasons, means for nitrogen schedules were not significant and highest cob girth was recorded with $\mathrm{T}_{5}$. Increase of such associated characters such as girth of cobs as a response to incremental $\mathrm{N}$ was previously reported by Gzazia et al., 2003, biofertilizer addition by Suke et al., 2010 and by application of Zn by Mohsin et al., 2014.

Table.1 Effect of nitrogen management schedules on vegetative and yield associated characters of maize cultivars.

\begin{tabular}{|c|c|c|c|c|c|c|c|c|c|c|}
\hline & \multirow{2}{*}{\multicolumn{2}{|c|}{$\begin{array}{l}\text { Plant Height } \\
(\mathrm{cm}) \\
(100 \text { DAS })\end{array}$}} & \multirow{2}{*}{\multicolumn{2}{|c|}{ DMA $\left(g / m^{2}\right)$}} & \multirow{2}{*}{\multicolumn{2}{|c|}{ LAI }} & \multicolumn{4}{|c|}{ Associated characters } \\
\hline & & & & & & & \multicolumn{2}{|c|}{$\begin{array}{l}\text { Ave length of } \\
\text { cobs }(\mathrm{cm})\end{array}$} & \multicolumn{2}{|c|}{$\begin{array}{l}\text { Average girth } \\
\text { of } \\
\text { cobs }(\mathrm{cm})\end{array}$} \\
\hline & $\begin{array}{l}2017- \\
2018\end{array}$ & $\begin{array}{l}2018- \\
2019\end{array}$ & $\begin{array}{l}2017- \\
2018\end{array}$ & $\begin{array}{l}2018- \\
2019\end{array}$ & $\begin{array}{l}2017 \\
- \\
2018\end{array}$ & $\begin{array}{l}2018- \\
2019\end{array}$ & $\begin{array}{l}2017- \\
2018\end{array}$ & $\begin{array}{l}2018- \\
2019\end{array}$ & $\begin{array}{l}2017- \\
2018\end{array}$ & $\begin{array}{l}\text { 2018- } \\
2019\end{array}$ \\
\hline \multicolumn{11}{|c|}{ Main Plot factor : Variety } \\
\hline $\mathbf{V}_{1}$ & 210.62 & 225.78 & 998.12 & 1079.96 & 3.03 & 3.15 & 15.85 & 16.32 & 12.88 & 13.24 \\
\hline $\mathbf{V}_{2}$ & 225.72 & 241.29 & 1008.35 & 1083.97 & 3.02 & 3.15 & 16.11 & 16.92 & 13.04 & 13.46 \\
\hline $\mathbf{V}_{3}$ & 183.59 & 196.99 & 880.00 & 943.36 & 2.40 & 2.48 & 14.84 & 15.14 & 12.55 & 12.75 \\
\hline Sem & 6.69 & 6.94 & 24.34 & 27.31 & 0.08 & 0.09 & 0.48 & 0.49 & 0.12 & 0.11 \\
\hline $\begin{array}{l}\text { CD } \\
(\mathbf{0 . 0 5})\end{array}$ & 26.96 & 28.02 & 98.26 & 110.26 & 0.34 & 0.38 & 1.93 & 1.99 & 0.49 & 0.46 \\
\hline \multicolumn{11}{|c|}{ Sub Plot : N management schedules } \\
\hline $\mathbf{T}_{1}$ & 176.99 & 189.38 & 699.39 & 678.41 & 2.11 & 2.05 & 14.07 & 14.54 & 12.86 & 13.03 \\
\hline $\mathbf{T}_{2}$ & 228.16 & 243.90 & 1050.82 & 1040.31 & 3.17 & 3.11 & 16.09 & 16.63 & 12.89 & 13.12 \\
\hline $\mathbf{T}_{\mathbf{3}}$ & 212.38 & 226.18 & 957.86 & 1033.20 & 2.71 & 2.81 & 15.87 & 16.40 & 12.56 & 12.84 \\
\hline $\mathbf{T}_{4}$ & 224.51 & 240.67 & 1038.20 & 1129.56 & 3.00 & 3.11 & 15.78 & 16.30 & 13.03 & 13.40 \\
\hline $\mathbf{T}_{5}$ & 235.27 & 255.03 & 1092.19 & 1192.67 & 3.27 & 3.41 & 16.61 & 17.18 & 13.75 & 14.11 \\
\hline $\mathbf{T}_{6}$ & 203.22 & 219.48 & 934.47 & 1026.05 & 2.62 & 2.73 & 15.19 & 15.70 & 11.84 & 12.40 \\
\hline Sem & 4.492 & 4.98 & 27.04 & 30.60 & 0.10 & 0.09 & 0.33 & 0.34 & 0.44 & 0.46 \\
\hline $\begin{array}{l}\text { CD } \\
(0.05)\end{array}$ & 13.03 & 14.46 & 78.44 & 88.74 & 0.30 & 0.28 & 0.95 & 0.98 & NS & NS \\
\hline
\end{tabular}

$\mathrm{V}_{1}$ :QPM (HQPM4); $\mathrm{V}_{2}$ : Shresta (hybrid) $\mathrm{V}_{3}$ : NAC 6004 (composite), $\mathrm{T}_{1}$ : Control ; $\mathrm{T}_{2}$ RDF; $\mathrm{T}_{3} \mathrm{RDN}_{75}+$ Vermi; $_{4}$ : $\mathrm{T}_{3}+\mathrm{Azo} ; \mathrm{T}_{5}: \mathrm{T}_{4}+\mathrm{Zn} ; \mathrm{T}_{6}: \mathrm{RDN}_{50}+\mathrm{Vermi}+\mathrm{Azo}+\mathrm{Zn}$ 
Table.2 Effect of nitrogen management schedules on yield parameters of maize cultivars.

\begin{tabular}{|c|c|c|c|c|c|c|c|c|c|c|c|c|}
\hline & \multicolumn{2}{|c|}{$\begin{array}{l}\text { No. of cobs/ } \\
\text { plant }\end{array}$} & \multicolumn{2}{|c|}{ No. of grains/ cob } & \multicolumn{2}{|c|}{$\begin{array}{l}\text { Average } 1000 \\
\text { grain weight of } \\
\text { cobs }(g)\end{array}$} & \multicolumn{2}{|c|}{$\begin{array}{l}\text { Mean weight of } \\
\text { grains/ cob }(\mathrm{g})\end{array}$} & \multicolumn{2}{|c|}{$\begin{array}{l}\text { Mean dry } \\
\text { weight of cob/ } \\
\text { plant (g) }\end{array}$} & \multicolumn{2}{|c|}{$\begin{array}{l}\text { Average } \\
\text { Shelling } \\
\text { percentage }(\%)\end{array}$} \\
\hline \multicolumn{13}{|c|}{ Main Plot factor : Variety } \\
\hline & $\begin{array}{l}2017- \\
2018\end{array}$ & $\begin{array}{l}\text { 2018- } \\
2019\end{array}$ & $\begin{array}{l}2017- \\
2018\end{array}$ & $\begin{array}{l}2018- \\
2019\end{array}$ & $\begin{array}{l}2017- \\
2018\end{array}$ & $\begin{array}{l}2018- \\
2019\end{array}$ & $\begin{array}{l}2017- \\
2018\end{array}$ & $\begin{array}{l}2018- \\
2019\end{array}$ & $\begin{array}{l}2017- \\
2018\end{array}$ & $\begin{array}{l}2018- \\
2019\end{array}$ & $\begin{array}{l}2017- \\
2018\end{array}$ & $\begin{array}{l}2018- \\
2019\end{array}$ \\
\hline $\mathbf{V}_{1}$ & 1.51 & 1.59 & 401.01 & 419.55 & 169.59 & 169.44 & 61.63 & 65.75 & 92.59 & 102.70 & 66.20 & 63.55 \\
\hline $\mathbf{V}_{2}$ & 1.52 & 1.60 & 374.35 & 389.79 & 175.78 & 176.72 & 65.96 & 70.34 & 96.38 & 106.07 & 68.22 & 66.11 \\
\hline $\mathbf{V}_{3}$ & 1.25 & 1.32 & 345.23 & 359.22 & 157.21 & 156.64 & 58.46 & 61.18 & 87.96 & 91.32 & 66.38 & 66.74 \\
\hline Sem & 0.02 & 0.02 & 6.50 & 6.24 & 0.96 & 0.97 & 1.14 & 1.16 & 0.70 & 0.69 & 1.36 & 1.39 \\
\hline $\begin{array}{l}\text { CD } \\
(0.05 \\
)\end{array}$ & 0.06 & 0.07 & 26.21 & 25.09 & 3.88 & 3.92 & 4.58 & 4.70 & 2.81 & 2.79 & NS & NS \\
\hline \multicolumn{13}{|c|}{ Sub Plot: N management schedules } \\
\hline $\mathbf{T}_{1}$ & 1.05 & 1.11 & 350.61 & 349.33 & 147.91 & 141.41 & 47.49 & 44.29 & 73.13 & 74.77 & 61.73 & 59.32 \\
\hline $\mathbf{T}_{2}$ & 1.49 & 1.57 & 394.16 & 399.64 & 176.46 & 176.64 & 69.39 & 71.66 & 98.94 & 102.76 & 70.25 & 69.74 \\
\hline $\mathbf{T}_{3}$ & 1.45 & 1.52 & 365.9 & 381.07 & 169.41 & 170.51 & 62.02 & 65.79 & 94.16 & 101.19 & 65.86 & 64.97 \\
\hline $\mathbf{T}_{4}$ & 1.49 & 1.57 & 386.93 & 409.87 & 178.33 & 179.03 & 68.82 & 72.90 & 100.39 & 106.77 & 68.16 & 68.24 \\
\hline $\mathbf{T}_{5}$ & 1.72 & 1.81 & 392.99 & 417.16 & 179.24 & 181.36 & 70.24 & 77.61 & 99.93 & 113.3 & 70.73 & 68.72 \\
\hline $\mathbf{T}_{6}$ & 1.37 & 1.45 & 350.60 & 380.04 & 153.81 & 156.64 & 54.14 & 62.28 & 87.32 & 101.37 & 64.86 & 61.79 \\
\hline Sem & 0.03 & 0.032 & 7.37 & 7.95 & 2.27 & 2.34 & 1.58 & 1.73 & 0.99 & 1.00 & 1.70 & 1.73 \\
\hline $\begin{array}{l}\text { CD } \\
(0.05 \\
)\end{array}$ & 0.09 & 0.011 & 21.40 & 22.83 & 6.60 & 6.73 & 4.59 & 4.98 & 2.85 & 2.89 & 4.93 & 4.99 \\
\hline
\end{tabular}

$\mathrm{V}_{1}$ :QPM (HQPM4); $\mathrm{V}_{2}$ : Shresta (hybrid) $\mathrm{V}_{3}$ : NAC 6004 (composite), $\mathrm{T}_{1}$ : Control ; $\mathrm{T}_{2}$ RDF; $\mathrm{T}_{3} \mathrm{RDN}_{75}+\mathrm{Vermi}_{4} \mathrm{~T}_{4}$ : $\mathrm{T}_{3}+\mathrm{Azo} ; \mathrm{T}_{5}: \mathrm{T}_{4}+\mathrm{Zn} ; \mathrm{T}_{6}: \mathrm{RDN}_{50}+\mathrm{Vermi}+\mathrm{Azo}+\mathrm{Zn}$ 
Table.2(a) Interaction of nitrogen management schedules and varieties on yield parameters of maize cultivars.

\begin{tabular}{|c|c|c|c|c|c|c|c|c|c|c|c|c|}
\hline & $\begin{array}{l}\text { No. } \\
\text { plant }\end{array}$ & cobs/ & $\begin{array}{l}\text { No. of } \\
\text { cob }\end{array}$ & grains/ & \multicolumn{2}{|c|}{$\begin{array}{l}\text { Average } 1000 \\
\text { grain weight of } \\
\text { cobs }(g)\end{array}$} & \multicolumn{2}{|c|}{$\begin{array}{l}\text { Mean weight of } \\
\text { grains/ cob (g) }\end{array}$} & \multicolumn{2}{|c|}{$\begin{array}{l}\text { Mean dry } \\
\text { weight of cob/ } \\
\text { plant }(\mathrm{g})\end{array}$} & \multicolumn{2}{|c|}{$\begin{array}{l}\text { Average } \\
\text { Shelling } \\
\text { percentage (\%) }\end{array}$} \\
\hline & $\begin{array}{l}2017- \\
2018\end{array}$ & $\begin{array}{l}2018- \\
2019\end{array}$ & $\begin{array}{l}2017- \\
2018\end{array}$ & $\begin{array}{l}2018- \\
2019\end{array}$ & $\begin{array}{l}2017- \\
2018\end{array}$ & $\begin{array}{l}2018- \\
2019\end{array}$ & $\begin{array}{l}2017- \\
2018\end{array}$ & $\begin{array}{l}2018- \\
2019\end{array}$ & $\begin{array}{l}2017- \\
2018\end{array}$ & $\begin{array}{l}2018- \\
2019\end{array}$ & $\begin{array}{l}2017- \\
2018\end{array}$ & $\begin{array}{l}2018- \\
2019\end{array}$ \\
\hline$V_{1} T_{1}$ & 1.20 & 1.26 & 379.14 & 384.31 & 146.54 & 138.86 & 40.39 & 37.86 & 68.24 & 67.56 & 59.41 & 56.05 \\
\hline$V_{1} T_{2}$ & 1.63 & 1.72 & 411.34 & 417.79 & 187.72 & 188.06 & 68.09 & 70.95 & 98.15 & 103.23 & 69.42 & 68.71 \\
\hline$V_{1} T_{3}$ & 1.51 & 1.57 & 376.11 & 391.15 & 163.81 & 164.1 & 59.56 & 65.54 & 92.62 & 105.10 & 64.46 & 62.39 \\
\hline $\mathbf{V}_{1} \mathbf{T}_{4}$ & 1.56 & 1.65 & 422.50 & 449.40 & 175.67 & 175.99 & 70.06 & 74.78 & 101.67 & 109.79 & 69.00 & 68.13 \\
\hline$V_{1} T_{5}$ & 1.77 & 1.84 & 429.90 & 452.10 & 186.11 & 186.44 & 70.58 & 79.21 & 100.55 & 117.59 & 70.25 & 67.39 \\
\hline$V_{1} T_{6}$ & 1.42 & 1.51 & 387.09 & 422.57 & 157.67 & 163.16 & 61.11 & 66.17 & 94.34 & 112.94 & 64.64 & 58.60 \\
\hline$V_{2} T_{1}$ & 1.06 & 1.12 & 354.82 & 346.24 & 153.81 & 149.73 & 54.63 & 50.76 & 84.67 & 87.61 & 64.60 & 57.91 \\
\hline $\mathbf{V}_{2} T_{2}$ & 1.58 & 1.68 & 398.71 & 404.66 & 175.98 & 176.68 & 70.12 & 71.42 & 100.01 & 104.44 & 70.11 & 68.36 \\
\hline$V_{2} T_{3}$ & 1.56 & 1.64 & 373.89 & 389.98 & 186.06 & 187.8 & 69.63 & 72.79 & 99.60 & 104.62 & 69.83 & 69.60 \\
\hline$V_{2} T_{4}$ & 1.56 & 1.65 & 373.92 & 397.01 & 193.52 & 195.69 & 72.38 & 77.48 & 101.03 & 108.10 & 70.27 & 71.70 \\
\hline$V_{2} T_{5}$ & 1.91 & 2.03 & 384.24 & 407.69 & 187.79 & 190.14 & 72.12 & 81.02 & 102.98 & 121.19 & 71.41 & 66.88 \\
\hline$V_{2} T_{6}$ & 1.42 & 1.51 & 360.52 & 393.14 & 157.53 & 160.26 & 56.91 & 68.58 & 90.00 & 110.45 & 63.09 & 62.19 \\
\hline$V_{3} T_{1}$ & 0.88 & 0.93 & 317.86 & 317.44 & 143.37 & 135.64 & 47.46 & 44.26 & 66.50 & 69.16 & 61.18 & 64.01 \\
\hline$V_{3} T_{2}$ & 1.27 & 1.32 & 372.42 & 376.48 & 165.68 & 165.18 & 69.95 & 72.61 & 98.67 & 100.62 & 71.22 & 72.14 \\
\hline$V_{3} T_{3}$ & 1.27 & 1.34 & 347.70 & 362.09 & 158.35 & 159.63 & 56.88 & 59.04 & 90.25 & 93.86 & 63.3 & 62.91 \\
\hline$V_{3} T_{4}$ & 1.34 & 1.41 & 364.37 & 383.21 & 165.81 & 165.42 & 64.02 & 66.45 & 98.49 & 102.43 & 65.21 & 64.90 \\
\hline$V_{3} T_{5}$ & 1.48 & 1.57 & 364.84 & 391.69 & 163.81 & 167.50 & 68.03 & 72.62 & 96.27 & 101.12 & 70.53 & 71.88 \\
\hline$V_{3} T_{6}$ & 1.27 & 1.34 & 304.19 & 324.40 & 146.23 & 146.49 & 44.40 & 52.09 & 77.61 & 80.71 & 66.87 & 64.58 \\
\hline & \multicolumn{2}{|l|}{ V X T } & \multicolumn{2}{|l|}{$\mathbf{V X T}$} & \multicolumn{2}{|l|}{ V X T } & \multicolumn{2}{|c|}{ V X T } & \multicolumn{2}{|l|}{ V X T } & \multicolumn{2}{|l|}{$\mathbf{V ~ X ~ T ~}$} \\
\hline Sem & 0.05 & 0.06 & 13.35 & 14.01 & 3.72 & 4.38 & 2.75 & 2.74 & 2.52 & 2.67 & 3.01 & 3.05 \\
\hline \multirow[t]{2}{*}{$\begin{array}{l}\text { CD } \\
(0.05)\end{array}$} & NS & NS & NS & NS & 11.09 & 13.11 & 8.50 & 8.20 & 7.54 & 8.01 & NS & NS \\
\hline & \multicolumn{2}{|l|}{ T X V } & \multicolumn{2}{|l|}{ T X V } & \multicolumn{2}{|l|}{ T X V } & \multicolumn{2}{|c|}{ T X V } & \multicolumn{2}{|l|}{ T X V } & \multicolumn{2}{|l|}{ T X V } \\
\hline Sem & 0.04 & 0.04 & 12.17 & 13.62 & 2.36 & 2.77 & 2.78 & 2.81 & 1.71 & 1.70 & 3.32 & 3.38 \\
\hline $\begin{array}{l}\text { CD } \\
(0.05)\end{array}$ & NS & NS & 60.54 & 63.24 & 11.73 & 12.89 & NS & NS & 7.94 & 7.89 & NS & NS \\
\hline
\end{tabular}

$\mathrm{V}_{1}$ :QPM (HQPM4); $\mathrm{V}_{2}$ : Shresta (hybrid) $\mathrm{V}_{3}$ : NAC 6004 (composite), $\mathrm{T}_{1}$ : Control ; $\mathrm{T}_{2} \mathrm{RDF} ; \mathrm{T}_{3} \mathrm{RDN}_{75}+\mathrm{Vermi}_{4} \mathrm{~T}_{4}$ : $\mathrm{T}_{3}+\mathrm{Azo} ; \mathrm{T}_{5}: \mathrm{T}_{4}+\mathrm{Zn} ; \mathrm{T}_{6}: \mathrm{RDN}_{50}+\mathrm{Vermi}+\mathrm{Azo}+\mathrm{Zn}$ 
Table.3 Effect of nitrogen management schedules on yield and quality attributes of maize cultivars

\begin{tabular}{|c|c|c|c|c|c|c|c|c|c|c|}
\hline & \multicolumn{2}{|c|}{ Grain yield (t/ha) } & \multicolumn{2}{|l|}{$\begin{array}{l}\text { Stover } \\
\text { (t/ha) }\end{array}$} & \multicolumn{2}{|l|}{$\begin{array}{l}\text { Harvest } \\
(\%)\end{array}$} & \multicolumn{2}{|c|}{$\begin{array}{l}\text { Protein content } \\
(\%)\end{array}$} & \multicolumn{2}{|c|}{$\begin{array}{l}\text { Carbohydrate } \\
\text { content }(\%)\end{array}$} \\
\hline \multicolumn{11}{|c|}{ Main Plot factor : Variety } \\
\hline & $\begin{array}{l}2017- \\
2018\end{array}$ & $\begin{array}{l}2018- \\
2019\end{array}$ & $\begin{array}{l}\text { 2017- } \\
2018\end{array}$ & $\begin{array}{l}2018- \\
2019\end{array}$ & $\begin{array}{l}2017- \\
2018\end{array}$ & $\begin{array}{l}2018- \\
2019\end{array}$ & $\begin{array}{l}2017- \\
2018\end{array}$ & $\begin{array}{l}2018- \\
2019\end{array}$ & $\begin{array}{l}2017- \\
2018\end{array}$ & $\begin{array}{l}2018- \\
2019\end{array}$ \\
\hline V 1 & 6.77 & 7.66 & 8.72 & 9.04 & 42.98 & 44.97 & 10.60 & 10.75 & 64.90 & 57.11 \\
\hline $\mathbf{V} 2$ & 7.19 & 8.26 & 8.88 & 9.20 & 44.09 & 46.60 & 7.77 & 7.81 & 66.12 & 66.82 \\
\hline V 3 & 5.26 & 5.82 & 7.68 & 8.11 & 40.08 & 41.24 & 7.13 & 7.05 & 62.47 & 62.61 \\
\hline Sem & 0.12 & 0.13 & 0.15 & 0.16 & 0.70 & 0.72 & 0.121 & 0.13 & 0.93 & 0.94 \\
\hline $\begin{array}{l}\text { CD } \\
(0.05)\end{array}$ & 0.48 & 0.55 & 0.59 & 0.66 & 2.84 & 2.91 & 0.49 & 0.51 & 3.75 & 3.81 \\
\hline \multicolumn{11}{|c|}{ Sub Plot: N management schedules } \\
\hline T 1 & 3.52 & 3.43 & 6.16 & 5.77 & 36.35 & 37.25 & 6.48 & 6.07 & 60.90 & 56.42 \\
\hline T 2 & 7.35 & 7.61 & 8.87 & 9.28 & 45.22 & 44.99 & 8.89 & 8.86 & 65.82 & 63.02 \\
\hline T 3 & 6.37 & 7.04 & 8.43 & 9.21 & 42.79 & 43.11 & 8.71 & 8.82 & 64.44 & 61.94 \\
\hline T 4 & 7.29 & 8.10 & 9.25 & 9.85 & 43.93 & 45.04 & 9.18 & 9.39 & 65.71 & 63.84 \\
\hline T 5 & 8.61 & 9.74 & 9.61 & 10.10 & 47.05 & 48.89 & 9.38 & 9.60 & 66.51 & 64.38 \\
\hline T 6 & 5.31 & 7.56 & 8.22 & 8.50 & 38.97 & 46.33 & 8.35 & 8.45 & 63.60 & 63.48 \\
\hline Sem & 0.21 & 0.22 & 0.17 & 0.20 & 0.96 & 1.02 & 0.11 & 0.12 & 1.27 & 1.30 \\
\hline $\begin{array}{l}\text { CD } \\
(0.05)\end{array}$ & 0.59 & 0.66 & 0.49 & 0.58 & 2.80 & 2.94 & 0.337 & 0.35 & N/S & N/S \\
\hline
\end{tabular}

$\mathrm{V}_{1}$ :QPM (HQPM4); $\mathrm{V}_{2}$ : Shresta (hybrid) $\mathrm{V}_{3}$ : NAC 6004 (composite), $\mathrm{T}_{1}$ : Control ; $\mathrm{T}_{2}$ RDF; $\mathrm{T}_{3} \quad \mathrm{RDN}_{75}+$ Vermi; $\mathrm{T}_{4}: \mathrm{T}_{3}+\mathrm{Azo} ; \mathrm{T}_{5}: \mathrm{T}_{4}+\mathrm{Zn} ; \mathrm{T}_{6}: \mathrm{RDN}_{50}+\mathrm{Vermi}+\mathrm{Azo}+\mathrm{Zn}$ 
Table.3(a) Interaction of nitrogen management schedules and varieties on yield and quality attributes of maize cultivars

\begin{tabular}{|c|c|c|c|c|c|c|c|c|c|c|}
\hline & \multicolumn{2}{|c|}{$\begin{array}{l}\text { Grain } \\
\text { (t/ha) }\end{array}$} & \multicolumn{2}{|c|}{$\begin{array}{l}\text { Stover } \\
\text { (t/ha) }\end{array}$} & \multicolumn{2}{|c|}{$\begin{array}{l}\text { Harvest } \\
(\%)\end{array}$} & \multicolumn{2}{|c|}{$\begin{array}{l}\text { Protein content } \\
(\%)\end{array}$} & \multicolumn{2}{|c|}{$\begin{array}{l}\text { Carbohydrate } \\
\text { content }(\%)\end{array}$} \\
\hline & $\begin{array}{l}2017- \\
2018\end{array}$ & $\begin{array}{l}\text { 2018- } \\
2019\end{array}$ & $\begin{array}{l}2017- \\
2018\end{array}$ & $\begin{array}{l}2018- \\
2019\end{array}$ & $\begin{array}{l}2017- \\
2018\end{array}$ & $\begin{array}{l}\text { 2018- } \\
2019\end{array}$ & $\begin{array}{l}2017- \\
2018\end{array}$ & $\begin{array}{l}\text { 2018- } \\
2019\end{array}$ & $\begin{array}{l}2017- \\
2018\end{array}$ & $\begin{array}{l}2018- \\
2019\end{array}$ \\
\hline $\mathbf{V}_{1} \mathbf{T}_{1}$ & 3.48 & 3.36 & 6.53 & 5.99 & 34.77 & 35.91 & 7.00 & 6.72 & 62.23 & 50.88 \\
\hline $\mathbf{V}_{1} \mathbf{T}_{2}$ & 7.93 & 8.06 & 9.18 & 9.53 & 46.35 & 45.8 & 11.34 & 11.45 & 65.56 & 56.46 \\
\hline $\mathbf{V}_{1} \mathbf{T}_{3}$ & 6.30 & 6.92 & 9.04 & 9.78 & 41.07 & 41.42 & 11.19 & 11.39 & 64.86 & 56.08 \\
\hline $\mathbf{V}_{1} \mathbf{T}_{4}$ & 7.81 & 8.43 & 9.64 & 10.22 & 44.76 & 45.25 & 11.83 & 12.10 & 66.14 & 59.23 \\
\hline $\mathbf{V}_{1} \mathbf{T}_{5}$ & 8.94 & 10.41 & 9.89 & 10.54 & 47.48 & 49.7 & 12.07 & 12.36 & 66.66 & 59.10 \\
\hline$V_{1} T_{6}$ & 6.18 & 8.74 & 8.03 & 8.15 & 43.49 & 51.72 & 10.19 & 10.44 & 63.97 & 60.94 \\
\hline $\mathbf{V}_{2} \mathbf{T}_{1}$ & 4.11 & 4.02 & 6.81 & 6.27 & 37.67 & 39.09 & 7.19 & 6.61 & 61.74 & 60.81 \\
\hline $\mathbf{V}_{2} \mathbf{T}_{2}$ & 7.84 & 8.18 & 9.31 & 9.65 & 45.71 & 45.88 & 7.84 & 7.92 & 67.45 & 68.80 \\
\hline $\mathbf{V}_{2} \mathbf{T}_{3}$ & 7.69 & 8.43 & 8.55 & 9.55 & 47.35 & 46.91 & 7.56 & 7.70 & 65.89 & 66.55 \\
\hline $\mathbf{V}_{2} \mathbf{T}_{4}$ & 7.99 & 9.00 & 9.47 & 10.12 & 45.76 & 47.09 & 8.15 & 8.34 & 67.77 & 68.45 \\
\hline $\mathbf{V}_{2} \mathbf{T}_{5}$ & 9.76 & 10.92 & 9.92 & 10.16 & 49.59 & 51.79 & 8.44 & 8.64 & 68.98 & 70.01 \\
\hline $\mathbf{V}_{2} T_{6}$ & 5.75 & 9.02 & 9.20 & 9.43 & 38.46 & 48.86 & 7.44 & 7.63 & 64.86 & 66.29 \\
\hline $\mathbf{V}_{3} \mathbf{T}_{1}$ & 2.97 & 2.92 & 5.14 & 5.03 & 36.62 & 36.75 & 5.25 & 4.88 & 58.74 & 57.57 \\
\hline $\mathbf{V}_{3} \mathbf{T}_{2}$ & 6.28 & 6.59 & 8.12 & 8.65 & 43.61 & 43.29 & 7.50 & 7.20 & 64.44 & 63.80 \\
\hline $\mathbf{V}_{3} \mathbf{T}_{3}$ & 5.12 & 5.76 & 7.70 & 8.29 & 39.94 & 41.00 & 7.38 & 7.38 & 62.57 & 63.20 \\
\hline $\mathbf{V}_{3} \mathbf{T}_{4}$ & 6.07 & 6.87 & 8.64 & 9.20 & 41.26 & 42.78 & 7.56 & 7.74 & 63.21 & 63.84 \\
\hline $\mathbf{V}_{3} \mathbf{T}_{5}$ & 7.12 & 7.89 & 9.03 & 9.59 & 44.09 & 45.19 & 7.63 & 7.81 & 63.89 & 64.02 \\
\hline$V_{3} T_{6}$ & 4.00 & 4.92 & 7.44 & 7.90 & 34.97 & 38.40 & 7.44 & 7.29 & 61.98 & 63.22 \\
\hline & \multicolumn{2}{|c|}{ V X T } & \multicolumn{2}{|c|}{ V X T } & \multicolumn{2}{|l|}{ V X T } & \multicolumn{2}{|l|}{ V X T } & \multicolumn{2}{|l|}{ V X T } \\
\hline Sem & 0.35 & 0.39 & 0.30 & 0.33 & 1.68 & 1.68 & 0.220 & 0.23 & 2.21 & 2.43 \\
\hline $\begin{array}{l}\text { CD } \\
(0.05)\end{array}$ & NS & 1.16 & NS & 0.98 & NS & NS & 0.713 & 0.73 & NS & NS \\
\hline & \multicolumn{2}{|c|}{ T X V } & \multicolumn{2}{|c|}{ T X V } & \multicolumn{2}{|l|}{ T X V } & \multicolumn{2}{|l|}{ T X V } & \multicolumn{2}{|l|}{$\mathbf{T} \mathbf{X} \mathbf{V}$} \\
\hline Sem & 0.39 & 0.43 & 0.36 & 0.39 & 1.72 & 1.72 & 0.29 & 0.31 & 2.27 & 2.36 \\
\hline $\begin{array}{l}\text { CD } \\
(0.05)\end{array}$ & 1.92 & 2.01 & NS & NS & NS & NS & 0.653 & 0.68 & NS & NS \\
\hline
\end{tabular}

$\mathrm{V}_{1}$ :QPM (HQPM4); $\mathrm{V}_{2}$ : Shresta (hybrid) $\mathrm{V}_{3}$ : NAC 6004 (composite), $\mathrm{T}_{1}$ : Control ; $\mathrm{T}_{2}$ RDF; $\mathrm{T}_{3} \mathrm{RDN}_{75}+\mathrm{Vermi}_{4} \mathrm{~T}_{4}$ : $\mathrm{T}_{3}+\mathrm{Azo} ; \mathrm{T}_{5}: \mathrm{T}_{4}+\mathrm{Zn} ; \mathrm{T}_{6}: \mathrm{RDN}_{50}+\mathrm{Vermi}+\mathrm{Azo}+\mathrm{Zn}$ 
Table.4 Mean Economics of different maize cultivars of 2017-18 and 2018-19 influenced by Nutrient management schedules

\begin{tabular}{|c|c|c|c|c|c|c|c|c|}
\hline & & $\mathbf{T}_{1}$ & $\mathbf{T}_{2}$ & $T_{3}$ & $\mathbf{T}_{4}$ & $T_{5}$ & $T_{6}$ & Mean \\
\hline \multirow[t]{2}{*}{$\mathbf{V}_{1}$} & $\begin{array}{l}\text { Net } \\
\text { Return } \\
\text { (Rs.) }\end{array}$ & $\begin{array}{l}30764.0 \\
0\end{array}$ & $\begin{array}{l}88109.0 \\
0\end{array}$ & $\begin{array}{l}53492.0 \\
0\end{array}$ & $\begin{array}{l}72548.0 \\
0\end{array}$ & $\begin{array}{l}86630.0 \\
0\end{array}$ & $\begin{array}{l}49214.0 \\
0\end{array}$ & $\begin{array}{l}63459.5 \\
0\end{array}$ \\
\hline & BCR & 1.92 & 2.99 & 1.27 & 1.67 & 1.91 & 1.15 & 1.82 \\
\hline \multirow[t]{2}{*}{$\mathbf{V}_{2}$} & $\begin{array}{l}\text { Net } \\
\text { Return } \\
\text { (Rs.) }\end{array}$ & $\begin{array}{l}46612.0 \\
0\end{array}$ & $\begin{array}{l}87712.0 \\
0\end{array}$ & $\begin{array}{l}72516.0 \\
0\end{array}$ & $\begin{array}{l}76106.0 \\
0\end{array}$ & $\begin{array}{l}99577.0 \\
0\end{array}$ & $\begin{array}{l}44708.0 \\
0\end{array}$ & $\begin{array}{l}71205.1 \\
7\end{array}$ \\
\hline & BCR & 2.91 & 2.98 & 1.72 & 1.76 & 2.20 & 1.05 & 2.10 \\
\hline \multirow[t]{2}{*}{$\mathbf{V}_{3}$} & $\begin{array}{l}\text { Net } \\
\text { Return } \\
\text { (Rs.) }\end{array}$ & $\begin{array}{l}31296.0 \\
0\end{array}$ & $\begin{array}{l}67126.0 \\
0\end{array}$ & $\begin{array}{l}37401.0 \\
0\end{array}$ & $\begin{array}{l}50321.0 \\
0\end{array}$ & $\begin{array}{l}63504.0 \\
0\end{array}$ & $\begin{array}{l}20704.0 \\
0\end{array}$ & $\begin{array}{l}45058.6 \\
7\end{array}$ \\
\hline & BCR & 2.23 & 2.45 & 0.93 & 1.22 & 1.47 & 0.51 & 1.47 \\
\hline \multirow[t]{2}{*}{ Mean } & $\begin{array}{l}\text { Net } \\
\text { Return } \\
\text { (Rs.) }\end{array}$ & $\begin{array}{l}36224.0 \\
0\end{array}$ & $\begin{array}{l}80982.3 \\
3\end{array}$ & $\begin{array}{l}54469.6 \\
7\end{array}$ & $\begin{array}{l}66325.0 \\
0\end{array}$ & $\begin{array}{l}83237.0 \\
0\end{array}$ & $\begin{array}{l}38208.6 \\
7\end{array}$ & \\
\hline & BCR & 2.35 & 2.81 & 1.31 & 1.55 & 1.86 & 0.90 & \\
\hline
\end{tabular}

$\mathrm{V}_{1}$ :QPM (HQPM4); $\mathrm{V}_{2}$ : Shresta (hybrid) $\mathrm{V}_{3}$ : NAC 6004 (composite), $\mathrm{T}_{1}$ : Control ; $\mathrm{T}_{2} \mathrm{RDF}_{3} \mathrm{~T}_{3} \mathrm{RDN}_{75}+\mathrm{Vermi}_{4}$ : $\mathrm{T}_{3}+\mathrm{Azo} ; \mathrm{T}_{5}: \mathrm{T}_{4}+\mathrm{Zn} ; \mathrm{T}_{6}: \mathrm{RDN}_{50}+\mathrm{Vermi}+\mathrm{Azo}+\mathrm{Zn}$

\section{Yield attributes}

Among the yield parameters shown in Table no.2, no. of cobs per plant is most contributory parameter for final yield. Shresta had 1.52 mean numbers of cobs and HQPM4 had 1.51 in 2017-18 and the corresponding values were 1.60 and 1.59 in 2018-19. Test weight varies little among varieties and the table reveals that Shresta enjoyed the highest mean test weight. The mean weight of grains per cob were highest for Shresta in both the seasons (65.96 $\mathrm{g}$ and $70.34 \mathrm{~g}$ respectively) with quite close performance of HQPM4 (61.63 g and $65.75 \mathrm{~g}$ respectively) having at par values.

The mean dry weight of cob was highest for Shresta (96.38 g and $106.07 \mathrm{~g}$ in respective seasons) which was significantly superior over
HQPM4 (92.59 $\mathrm{g}$ and $102.70 \mathrm{~g}$ in respective years) and the improvement in cob weight in the $2^{\text {nd }}$ year implies the compounding effect of organic sources, such findings were also made by Zhang et al., 2016. Mean number of grains per cobs in HQPM4 were significantly higher 401.01 and 419.55 in the successive years. Varying performance of maize varieties in grain yield was reported by Assaduzzaman et al., 2014.

Among the nutrient schedules $\mathrm{T}_{5}$ had the highest mean number of cobs (1.72 in 201718 and 1.81 in 2018-19). Also highest number of grains per cob at par with $\mathrm{T}_{5}$ (392.99) and $\mathrm{T}_{4}$ (386.93) in 2017-18, highest number of grains per cob, test weight in the successive years, grain weight per cob (70.24 g in 201718 and $77.61 \mathrm{~g}$ in 2018-19) and mean dry 
weight of cobs along with implied shelling \% (70.73\% in $17-18$ and $69.74 \%$ in $18-19)$ were significantly higher in $\mathrm{N}$ management schedules of $75 \% \quad \mathrm{~N}$ along with vermicompost, Azotobactor and $\mathrm{Zn}$ conjunction. Effect of INM coupled biofertilizer sources and $\mathrm{Zn}$ was also reported to be better in maize by Khan et al., 2008, Ram et al., 2009 on INM -N sources by Shinde et al., 2011 and numerous other workers.

\section{Yield}

Shresta had the highest significant yield of 7.19 t/ha in 2017-18 and was at par with Improvement of stover yield by $\mathrm{N}$ administration through compost was reported by Shinde et al., 2011 and Khan et al., 2008. Biofertilizer application significantly improved stover yield in experiments conducted by Balyan et al., 2006.

In both the seasons, among the varieties, Shresta performed the best with the harvest index of $44.09 \%$ and $46.60 \%$ respectively which were at par with HQPM4 (42.98\% and $44.97 \%$ ) with HI of both were superior and significant over NAC 6004 maize type in both the seasons. The management schedule which proved to be the best was $\mathrm{T}_{5}$, enjoying a mean HI of $47.05 \%$ and $48.89 \%$ in the respective years. The interaction values for HI were not significant. Mohsin et al., 2014 also reported highest harvest index by application of zinc.

\section{Quality Attributes}

HQPM4 had the significantly higher protein content of $10.60 \%$ and $10.75 \%$ in 2017- 18 and 2018-19 respectively while Shresta had significantly higher carbohydrate content of in the respective years, keeping two other cultivars far behind. $\mathrm{N}$ management schedules $75 \% \mathrm{~N}$ along with vermicompost, azotobactor and zinc had higher protein content of $9.38 \%$
HQPM4 (6.77 t/ha) while in the $2^{\text {nd }}$ year it was significantly higher than HQPM4 (7.66 t/ha). $\mathrm{RDN}_{75}+$ Vermicompost @ 2 t/ha + Azotobactor@2kg/ha+ Zn was the best nutrient schedule and it performed best with Shresta, hybrid maize (9.76 t/ha in 2017-18 and $10.92 \mathrm{t} / \mathrm{ha}$ in 2018-19), integrated sources using compost was reported with greater grain yield by Rajasingh et al., 2014. The stover yield of both the hybrid cultivars (HQPM4 and Shresta) were at par in both the seasons. In both the seasons among the management schedules $T_{5}$ performed the best with $9.61 \mathrm{t} / \mathrm{ha}$ and $10.10 \mathrm{t} / \mathrm{ha}$ of stover yield respectively.

and $9.60 \%$ in respective years. Findings of Ram et al., 2009 corroborates that organic sources result in greater grain protein content and Karki et al., 2005, observed similar findings. Balai et al., 2011 observed improved carbohydrate in maize with application of compost sources. Comparable mean protein content of QPM has been supported by literature (Alamerew, 2008).

The mean maximum net return was obtained by variety Shresta (Rs. 71205.17/-) and mean BCR was 2.10 which was followed closely by HQPM4 (Table No. 4). Among the nutrient schedules the maximum net return was registered by $\mathrm{RDN}_{75}+$ Vermicompost @ 2 t/ha+Azotobactor@2kg/ha+ Zn amounting to Rs. 83237.67/- with a corresponding mean BCR of 1.86. The combination of the above treatments $\left(\mathrm{V}_{2} \mathrm{~T}_{5}\right)$ achieved a BCR of 2.20 and net revenue of (Rs. 99577/-) per hectare.

Among three maize cultivars hybrid Shresta may be recommended for the eastern plains and HQPM4 also enjoys good production potential. It is also concluded that inorganic fertilizer $\mathrm{N}$ can be discounted to the extent of $25 \%$ and $\mathrm{RDN}_{75}+$ Vermicompost @ 2 t/ha + Azotobactor@2kg/ha+ Zn with respective economics of 1.86. The economics was 
favoured for the single hybrid maize, Shresta with a value of $2.10 \mathrm{BCR}$.

\section{Acknowledgement}

Authors are very thankful to the Department of Agronomy, Faculty of Agriculture, Bidhan Chandra Krishi Viswavidyalaya, Nadia, West Bengal for catering all the necessary facilities and support for the successful experimentation.

\section{References}

Alamerew, S. 2008. Protein, tryptophan and lysine content in Quality Protein Maize, North India.

2008. Ethiop J Health Sci., 18(2): 9-14.

Allah, W., Tahir, Muhammad., Manaf, Abdul., Ahmed, M., Kaleem, S., Ahmad, I. 2011. Improving maize productivity through tillage and nitrogen management. African Journal of Biotechnology, 10 (82): 19025-19034.

Anjorin, F. B. 2013. Comparative Growth and Grain Yield Response of Five Maize Varieties to Nitrogen Fertilizer Application. Greener Journal of Agricultural Sciences, ISSN: 22767770, ICV: 6.15, 3 (12), pp. 801-808.

Arya, K.C., Singh, S.N. 2000. Effect of different levels of $\mathrm{P}$ and $\mathrm{Zn}$ on yield and nutrient uptake of maize (Zea mays L.) with and without irrigations. Indian J. Agron., 45(4): 717-721.

Asaduzzaman, M., Biswas, M., Islam, N., Rahman, M. M., Begum, R., Rahman, M. A. 2014. Variety and N-Fertilizer Rate Influence the Growth, Yield and Yield Parameters of Baby Corn (Zea mays L.). Journal of Agricultural Science, 6(3), ISSN 1916-9752 EISSN 1916-9760.

Balai, M. L., Arvind, V., Nepalia, V., Kanthaliya. 2011. Productivity and quality of maize (Zea mays L.) as influenced by integrated nutrient management under continuous cropping and fertilization. Indian $\mathrm{J}$. Agric. Sci., 81: 374-376.

Balyan, J. K., Singh, P., Jain, L. K., Jat, M. L. 2006. Maize (Zea mays L.) productivity in response to integrated nutrient management in southern Rajasthan. Curr.Agric.Curr. Sci., 13: 129-132.

Dadhich, S. K., Somani, L. L., Shilpkar, D. 2011. Effect of integrated use of fertilizer P, FYM and biofertilizers on soil properties and productivity of soybean-wheat crop sequence. Adv. Dev. Res., 2: 42-46.

Das, A., Raychaudhuri, U., Chakraborty, R. 2012. Cereal based functional food of Indian subcontinent: a review. J. Food Sci Technol., 49(6):665-72. doi: 10.1007/s13197-011-0474-1.

Dhoke, N. R., Dahatonde, B. N., Thakur, M. R., Ulemale, R. B. 2007. Soil nutrient balance, its physico-chemical properties and yield of maize as influenced by integrated nutrient management. Crop Prot. Prod. J., 4(1): 21-23.

FAO statistical yearbook. 2013. Feeding the world. Pp 130.

FAO FOOD AND NUTRITION PAPER 77. 2003. Report of a Technical Workshop, Rome, In Food energy methods of analysis and conversion factors.

Food and Agriculture organization Of United Nations, Rome, Italy, 1995. Dimensions of need, An atlas of food and agriculture.

Gunaratna, N. S., Moges, D., Groote, H. D. 2019. Biofortified maize can improve quality protein intakes among young children in Southern Ethiopia. Nutrients, 11(1): 192 . doi: 10.3390/nu11010192.

Gzazia, J. D., Tittonell, P.A., Germinara, D., 
Chiesa, A. 2003. Phosphorus and nitrogen fertilization in sweet corn (Zea mays L. saccharata). Spanish J. Agric. Res., 1(2): 103-107.

Jackson, M. L. 1973. Soil Chemical Analysis, Prentice Hall of India Private Limited, New Delhi, pp.498.

Karki, T. B., Kumar, A., Gautam, R. C. 2005. Influence of integrated nutrient management on growth, yield, content and uptake of nutrients and soil fertility status in maize (Zea mays L.). Indian J. Agric. Sci., 75(10): 682-685.

Khan, H. Z., Malik, M. A., Saleem, M. F. 2008. Effect of rate and source of organic material on the production potential of spring maize (Zea mays L.). Pakistan J.Agric. Sci., 45(1): 4043.

Kizilkaya, R. 2008. Yield response and nitrogen concentration of spring wheat inoculated with

Azotobacter chroococcum strains. Ecol. Engg., 33: 150-156.

Kumar, A., Rajgopal, D. S., Kumar, L. 2008. Effect of vermicompost, poultry manure and azotobactor inoculation on growth, yield and nutrient uptake of sweet corn. Indian J. Agron., 34(4): 342- 347.

Kumari, M., Singh, O.P., Meena, D. C. 2017. Crop Water Requirement, Water Productivity and Comparative Advantage of Crop Production in Different Regions of Uttar Pradesh, India, International Journal of Current Microbiology and Applied Sciences, ISSN: 2319-7706, 6 (7) 2043-2052. http://dx.doi.org/10.20546/ijcmas.2017 .603.242.

Lal, B., Singh, D. 1998. Crop yield and uptake of potassium by maize, wheat and cowpea fodder in relation to various potassium forms in soil under intensive cropping and continuous fertilizer use. Indian J. Agric. Sci., 68(1): 754-755.
Mohsin, A. U., Ahmad, A. U. H., Farooq, M., Ullah, S. 2014. Influence of zinc application through seed treatment and foliar spray on growth, productivity and grain quality of hybrid maize. Journal of Animal and Plant Sciences, 24(5): 1494-1503

Mukherjee, A., Wang, S. Y. S., Promchote, P. 2019. Examination of the climate factors that reduced wheat yield in Northwest India during the 2000s. Water, $\quad 11(2)$ : 343 . https://doi.org/10.3390/w11020343.

Murdia, L. K., Wadhwani, R., Wadhawan, N., Bajpai, P., Shekhawat, S. 2016. Maize Utilization in India: An Overview. American Journal of Food and Nutrition, 4(6) 169-176. http://pubs.sciepub.com/ajfn/4/6/5 (C)Science and Education Publishing.doi:10.12691/ajfn-4- 65,2016.

Prasanna, B. M., Vasal, S. K., Kassahun, B., Singh, N. N. 2001. Quality protein maize. Current Science, 81(10): 13081319.

Rajasingh, R. S., Lourduraj, A. C. 2014. Effect of integrated nutrient management on yield attributes and yield of maize hybrid. International Journal of Agricultural Sciences. 10(2): 761-765

Ram, P., Solanki, N. S., Singh, D., Dadheech, R. C. 2009. Growth, yield and economics of quality protein maize (Zea mays L.) as influenced by fertility levels and foliar spray of thiourea. Haryana Journal of Agronomy, 25(1/2): 73-75.

Savalgi, V. P., Savalgi, V. 1992. Effects of Azospirillum brasilense and earth warm cast as seed treatment on sorghum. J. Maharashtra Agric. Univ., 16: 107-108.

Sheoran, O.P., Tonk, D.S., Kaushik, L.S., Hasija, R.C., Pannu, R. S. 1998. 
Statistical Software Package for Agricultural Research.

Shinde,P. D., Jadhav, A. S., Shaikh, A. A. 2011. Effect of integrated nutrient management and row spacings on growth and yield of composite maize (Zea mays L.). Journal of Maharashtra Agricultural Universities, 36(1): 134137.

Suke, S. N., Deotale, R. D., Hiradeve, P., Deogirkar, M., Sorte, N. V. 2010. Effect of nutrients and biofertilizer on yield and yield contributing parameters of maize (Zea mays L.). J. Soils and
Crops, 20 (2): 278-283.

Watson, D.J. 1947. Comparative physiological studies in the growth of field crops. I. Variation in net assimilation rate and leaf area between species and varieties, and within and between years. Annals of Botany, 11: 41-76.

Zhang, Y., Li, T., Wu, H., Bei, S., Zhang, J., $\mathrm{Li}, \quad \mathrm{X}$. 2019. Effect of different fertilization practices on soil microbial community in a wheat-maize rotation System. Sustainability, 11, 4088, doi:10.3390/su11154088.

\section{How to cite this article:}

Ananya Chakraborty, Sritama Biswas, Rajarshi Banerjee, Pintoo Bandopadhyay and Srijani Maji 2019. Differential Response of QPM, Hybrid and Composite Maize Cultivars to INM Schedules. Int.J.Curr.Microbiol.App.Sci. 8(09): 2142-2154.

doi: https://doi.org/10.20546/ijcmas.2019.809.248 